Daimon. Revista Internacional de Filosofía, Suplemento 5 (2016), 757-763

ISSN: 1130-0507 (papel) y 1989-4651 (electrónico)

http://dx.doi.org/10.6018/daimon/268881

\title{
Utopología o las alternativas a la fragmentación de la subjetividad
}

\author{
Utopology, or, alternatives \\ to subjectivity fragmentation
}

ABRAHAM RUBÍN*

\begin{abstract}
Resumen: En este artículo planteamos la necesidad de desarrollar un análisis de las tendencias de resistencia al proceso de mercantilización, aunque este tenga lugar a través de formas distorsionadas -incluso inconscientes-, que proyectan las tentativas de la imaginación para salir de una parálisis que lleva consigo la imposibilidad de pensar una alternativa al sistema que no pase por una fase catastrófica, de total destrucción. Este estudio de las proyecciones de futuros alternativos, podría denominarse, siguiendo la intuición de Fredric Jameson y en línea con el concepto de genealogía (del pasado), una utopología. Postulamos que este trabajo puede ayudar a reconvertir la fragmentación de la psique y sus derivas corporales en una operación útil para la intervención política.
\end{abstract}

Palabras llave: Utopía, mercantilización, Jameson, subjetividad.

\begin{abstract}
This article raised the question of developing an analysis of resistances to commodification process, even through unconscious distorted forms, which project attempts of imagination to free itself from an imaginative paralysis that carries inability to think an alternative system that does not pass through a catastrophic phase of mass destruction. This study of alternative futures projections could be called, following Fredric Jameson's intuition and in the same way of the concept of genealogy, an utopology. We postulate that this work can help to transform the psyche fragmentation into a useful policy operation.
\end{abstract}

Keywords: Utopia, commodification, Jameson, subjectivity.

La cuestión de la utopía nos sale al paso siempre que nos planteamos alternativas al respecto de un momento histórico. En el caso actual, aparece como una (im)posible respuesta a la que creemos es la fragmentación de la subjetividad con la que el capitalismo atenaza a los cuerpos, a través de la paralización y prefabricación del deseo con la que actúa el creciente proceso de mercantilización de la vida, caballo de batalla de toda reflexión acerca de las herencias del neoliberalismo.

Fecha de recepción: 02/06/2016. Fecha de aceptación: 17/07/2016.

* Profesor de enseñanza secundaria de la Comunidad de Madrid. Líneas de investigación: Postestructuralismo, postmodernidad, postmarxismo, deconstrucción. Recientemente ha publicado la obra Vivir el acontecimiento. Aproximaciones desde el pensamiento contemporáneo. Santiago de Compostela, Universidad de Santiago de Compostela Editora, 2016; y, en lengua gallega, el ensayo Corpo-circuítos. Máquinas de subxectivación. Vigo, Euseino? Editores, 2016. Correo electrónico: abrahamrubinalvarez@gmail.com 
La economía pasa a ser el ámbito dominante del poder y la esfera semiótica general. De ese modo las subjetividades gobernadas bajo la acción del biopoder se convierten en figuras que padecen la bioeconomía, término popularizado por Andrea Fumagalli para dar cuenta de las consecuencias de la centralidad del mercado en tanto lugar en el que las relaciones de poder se manifiestan y redefinen continuamente (Fumagalli, 2010,26). Con la bioeconomía entramos en la valorización en términos exclusivamente económicos de la vida misma. Es el ámbito de acción consecuencia de la multiplicación del homo economicus.

El estadio económico del capitalismo que se va a ocupar de desarrollar este particular biopoder es el que en la corriente de pensamiento postoperaista italiano se conoce con el nombre de capitalismo cognitivo, término que pretende mostrar que los nuevos territorios de explotación económica se refieren a las capacidades vitales del ser humano, como por ejemplo el lenguaje y el conocimiento. Desde esta visión, las materias primas más importantes que forman parte de una subjetividad -al producirla o conformarla- no son las físicas, sino el saber, la inteligencia o los afectos: las cualidades cognitivo-inmateriales (Marazzi, 2014).

En todo caso, a través del concepto de bioeconomía podemos entender mejor el paso a la sociedad de autocontrol en el que las subjetividades son producidas a partir del sistema económico. Si, efectivamente, no puede pensarse una sociedad sin control -orden, normas-, el autocontrol se refiere a todo aquello que ya no viene impuesto, sino que pasa a estar interiorizado, formando parte de aquel que lo porta. De esa manera, la cuestión ya no es si el control surge de un pacto social o de una imposición, sino de qué manera esta cuestión permanece olvidada en la (re)producción de la subjectividad, que pretende desactivar la posibilidad de salirse de la herencia recibida -sea esta un pacto libre o impuesto-.

Para seguir esta línea tenemos que situarnos en una perspectiva que corresponda a las grandes formaciones, es decir, las estrategias de subjetivación a gran escala. Pues son las grandes esferas de poder las que implantan una política económica que sirve como base sobre la que se modela la política estatal, llevando consigo a su vez una subjetivación económica de la ciudadanía hecha a partir de los intereses de aquellas.

Como decíamos, el punto de partida es la posición central del mercado, después de afianzarse como tal a partir del gobierno liberal. Desde nuestra perspectiva el mercado no es un lugar neutral en el que los individuos intercambian objetos libremente -como es la visión asumida por el liberalismo- sino, al contrario, un espacio cerrado y controlado por un pequeño número de agentes económicos a la búsqueda de la satisfacción de sus intereses particulares. Esta perspectiva se confirma con la aparición de los mercados financieros y la financiarización de la economía mundial.

Más allá de hacer una reconstrucción histórica de los motivos que llevaron a esta realidad actual incuestionable (para la cual se puede consultar, por ejemplo, Arrighi, 1994, Marazzi, 1998 y 2014, o Fumagalli, 2010), lo que nos interesa es ver cómo la nueva ola de fabricación de subjetividades se realizó aprovechando la multiplicación de la participación de los ciudadanos en los mercados financieros. A partir de la financiarización del Estado se hizo depender de las cotizaciones bolsistas buena parte de la renta y de las seguridades con que el Estado de bienestar protegía a los ciudadanos, tales como los planes de pensiones o fondos de inversión. Ahora ya no es el Estado el que tiene la capacidad autónoma de coaccionar de una u otra forma a los trabajadores porque ese papel es cumplido eficientemente por un 
agente supuestamente neutral, el mercado financiero. Por ello, al depender la vida de los ciudadanos del momento que vivan los mercados bolsistas nos encontramos con una forma de control férreo sobre las subjetividades, que se desarrollan con unas u otras condiciones en función del momento vivido por la economía. Ya no estamos más en la discusión acerca de si el mercado se debe o no controlar por el Estado, sino que es él lo que pasa a ser el controlador de toda política. De esa manera la subjetividad asume que el mercado es un lugar objetivo, de veridicción, y que sobre su base se deben juzgar tanto las acciones del Estado como las suyas propias en tanto trabajador o ciudadano.

Sin duda uno de los ejemplos más determinantes es el creciente papel de los planes de pensiones, donde se impulsa la construcción subjetiva en una doble vertiente. En primer lugar, se subjetiva al ciudadano para adaptarlo a las condiciones del mercado financiero y que salga fuera de la protección del Estado de bienestar. Es decir, se pretende que los ciudadanos se acostumbren a su próxima desaparición. Es una subjetivación del riesgo, en cuanto identifica como algo normal el hecho de asumir los riesgos históricamente asociados con el Estado. En segundo lugar, se inserta el ahorro de los ciudadanos en los mercados. Esto es fundamental para relacionar los procesos de transformación capitalistas con el ahorro de los trabajadores, introduciendo su capacidad de ahorro en el juego financiero. De ese modo se consigue modificar el déficit de los Estados, a través de la inversión en Bolsa de parte de lo ahorrado (Lazzarato, 2013, 45).

Un factor decisivo en los mercados financieros es el psicológico, aquel que hace creer a los inversores en determinado momento que unos títulos están a punto de aumentar su valor. Esa transmisión de información no se basa, mayoritariamente, en una serie de especialistas que estudian a fondo las sociedades que cotizan en bolsa, sino en una técnica más bien intuitiva que trata de percibir qué títulos van a ser los depositarios de la confianza de los inversores, para unirse a tiempo al grupo, generando un comportamiento imitativo por parte de otros inversores que provoca que el grupo $-y$ la burbuja que se forma a su alrededor- sea cada vez más grande. Este comportamiento, además, conlleva que el mercado se vea como un lugar democrático, donde cualquiera puede hacer fortuna -visión clásica de Wall Street-. Desde ese punto se potencia el individualismo que parte de un supuesto agente económico solitario que puede enriquecerse gracias a sus aptitudes. Asimismo facilita la visión autorreferencial del mercado, teniendo solo en cuenta el modo de actuar de los inversores y no un supuesto valor económico objetivo externo al mismo (Fumagalli, 2010, 64).

Con la misma lógica, cuando el mercado no funciona, cuando la sociedad y el mercado se desajustan en algunos aspectos, provocando sufrimiento en la población, contra toda evidencia lo que se propone es que la sociedad se ajuste al mercado, corriendo los riesgos y esfuerzos que sea menester, incluso si ello significa la revuelta o la disgregación de la población. Pero este funcionamiento produce una determinada carga semiótica, subjetivaciones de las que es difícil salir, signos que una subjetividad reconoce como propios de la riqueza y a los cuales se adhiere, terminando de completar el proceso de producción de subjetividad iniciado por las técnicas de comunicación.

Fumagalli recuerda un cuento de Walt Disney en el que Scrooge McDuck -conocido como Rico McPato o Tío Rico en Hispanoamérica, y Gilito McPato o Tío Gilito en Españaes obligado por su enemigo a comprar acciones de una empresa al borde de la quiebra, con 
la intención de arruinarlo. Sin embargo, cuando la noticia se hace pública, una multitud de individuos se lanzan a comprar acciones de dicha compañía, seguros de la intuición y de los motivos fiables que guían a Scrooge McDuck a tal inversión, ayudando con su participación a reflotar a la compañía. De esta manera McDuck no solo no se arruina sino que se enriquece aún más con una buena cantidad de dinero con la que no contaba (Ibid.). Dicho de otra forma, las estrategias comunicativas de las sociedades de inversión transforman su opinión en una referencia, en la medida que convence a más o menos público, llegando a ser considerada entonces “opinión pública". La producción de enunciados realizada por los mercados es la semiótica dominante y, al mismo tiempo, la adhesión a ella de las subjetividades muestra la idea de que la subjetivación bioeconómica es la clave del papel predominante de los mercados financieros en la actualidad.

La utopía, por el contrario, responde a la figura de la totalidad -al igual que el capitalismo- de dos maneras distintas, entendiéndola en tanto sistema y como anticipación de todo cambio futuro guiado por el deseo humano. Mas con utopía no nos referimos aquí a ningún idealismo, ni siquiera a un ideal regulativo de tipo kantiano, sino a la forma y (no)lugar en donde creemos que sobrevive el deseo en la actualidad, después de que el capitalismo ejerza como idea sistémica y como paralizador de la imaginación humana, cortocircuitándola y redireccionándola a través del consumo y la idea de mercado.

La primera de las posibilidades mencionadas se refiere a la figuración de la totalidad como la idea de sistema, donde todo está conectado, donde las anomalías que surgen y que no se ajustan al sistema solamente lo descuadran temporalmente, mientras este se reajusta, incorporando las anomalías a su funcionamiento estándar. Desde esa visión se puede interpretar el modo de operación habitual del capitalismo, deviniendo por tanto una figura distópica, que dota de sentido significante a toda la realidad -subjetividad paranoica-.

Al mismo tiempo, la generalización del funcionamiento basado en el consumo de mercancías, y en la disponibilidad de un número ingente de ellas esperando para ser compradas y consumidas, lleva al deseo a un bucle reactivo sin capacidad creativa. Todo está disponible, el deseo se prefabrica, la imaginación se paraliza y se atrofia la capacidad de desear.

Por el contrario, la utopía entiende la totalidad en tanto acontecimiento, es decir, como acción transformadora de las subjetividades (incluso como conformación colectiva extrahumana de la subjetividad). La utopía, a través de la acción del deseo humano, sería entonces la anticipación de todo cambio llegado con el porvenir. El deseo, en muchas ocasiones no es consciente de aquello a lo que apunta, o trabaja mediante distorsiones o fragmentos discontinuos. Eso lo hace perder efectividad, pero no provoca su desaparición. Sigue operando en la utopía de lo cotidiano, introduciendo la posibilidad (imposible) del desarrollo de lo real. Siempre que salimos fuera de nuestra mismidad para abrirnos a lo otro, lo imposible deviene la introducción a aquello posible (en tanto es imposible), su condición de posibilidad (Derrida, 2003, 249-280). De aquí la necesidad que vemos de promover subjetivaciones utópicas que ayuden a catalizar el deseo del deseo, un aprender de nuevo a desear fuera de vías consumistas para abrir un espacio que pueda dar lugar a una conformación de la subjetividad más allá de las semióticas dominantes.

El capitalismo se apoya en lo que se podría denominar un proceso de taylorización mental, donde una de sus consecuencias es la fragmentación de la psique, y con ella, de la subjetividad. Los modelos semióticos dominantes centran parte de sus intereses en la 
tentativa de potenciar el desarrollo por parte de la población de las áreas mentales que se ocupan del cálculo o la aritmética, es decir, aquellas que pueden ser más útiles -y rentables-. Estas se alejan poco a poco de aquellas áreas más creativas -y menos útiles-, como la imaginación, llevando consigo la parálisis del deseo con la que se enfrenta la utopía.

La utopía focaliza su interés en la colectividad, y desde ahí pretende construir algo diferente a lo actual. Pues uno no puede salir del candado semiótico del capital de modo individual -sino a través de conformaciones plurales, colectivas y/o metahumanas-, ni huir de los rastros que la identidad deja alrededor de toda tentativa imaginativa (anticipación de un presente-futuro). Pero más allá de la concepción de la utopía como construcción, existe todo un haz de deseos desarticulados y desorganizados -a veces ingenuos-, pero que muestran o dejan entrever una resistencia al proceso de mercantilización de la vida. Estas resistencias se detectan en lo cotidiano, en aquello que Ernst Bloch llamaba impulso (utópico) (Bloch, 1977, 32-145; Jameson, 2009, 15-24), pues «en el presente [...] hay un impulso y una ruptura, una incubación y una anticipación de aquello que todavía no ha llegado a ser» (Bloch, 1977, 9). Estos impulsos son fragmentos distorsionados, incluso inconscientes, que esconden nuevos deseos que esperan para tomar forma. Solo a partir de ahí es posible el surgimiento de algo diferente, de una subjetividad alternativa.

Parece importante, en ese sentido, destacar el papel que a nuestro juicio ha cumplido el texto utópico (y la literatura de ciencia ficción, en general) en tanto cristalizadores del deseo y el acontecimiento desde una perspectiva histórica, ya que la literatura siempre está enraizada en las actitudes sociales de su tiempo. Pues el texto utópico presenta la propia sociedad del escritor con sus hábitos rituales inconscientes traspuestos a sus equivalentes conscientes (Frye, 1982, 57).

El intento del texto utópico es intentar crear algo a partir del material con el que se cuenta en un presente, es decir, repetir diferencialmente una situación irrepetible o dar cuenta de la herencia que la propia utopía recibe, la herencia del aquí y ahora. El salto utópico es el precio exigido para poder imaginar la utopía. Pues ella nos desborda, nos sobrepasa. De aquí que el salto vuelva el tiempo y/o el espacio distinto, con las pautas sociales derivadas del mismo también presentes. Así, la ciencia ficción (y la utopía) se pueden entender narrativamente anclados en el concepto de novum, una novedad o innovación que presenta en la narración una relación totalizadora que se desvía de la norma de realidad del autor y del lector, produciendo en ellos un extrañamiento cognitivo -metamorfosis del concepto brechtiano- (Suvin, 1984, 25-29) que los libera de las cadenas de su presente y de la mismidad cerrada en la que este tiene lugar. El correlato del novum es una realidad alternativa, con un tiempo histórico diferente, pero no pretende hacer predicciones sobre el futuro -eso no sería más que desarrollar lo posible implicado en lo real-, sino reflexionar sobre el período histórico del autor y aquello que lo amenaza desde la no presencia (Ibid., 102-109).

Cuando en la novela Ubik el personaje Joe Chip tiene que introducir monedas para abrir la puerta del frigorífico -incluso el de su casa- no es porque Philip K. Dick esté pronosticando el futuro, sino constatando críticamente su presente -transcurriendo la acción, eso sí, en un apartamento del futuro-. La herramienta clave para conseguir el extrañamiento cognitivo es el novum; en el caso de Ubik es el extraño producto con el cual uno puede controlar su destino -y que da nombre a la novela-; en Foundation de Isaac Asimov es la 
psicohistoria; en Solaris de Lem es el océano de plasma, mientras que en su Kongres futurologiczny es la psiquímica, etcétera. En esa línea trabajan también las proyecciones de los impulsos utópicos.

Todos ellos son fragmentos desorganizados e incoherentes, pero denotan el deseo escondido tras ellos: la reconfiguración y conformación de circuitos donde este pueda fluir, y que precisan del otro, un otro que no solo es humano, sino también maquinal, económico, social o tecnológico. Un otro que forma parte de un proceso colectivo, más allá de lo individual, formando una dimensión extrapersonal.

En todo caso, intervenir en el circuito semiótico es una de las tareas a asumir por un trabajo de producción semiótica alternativa, que pretenda huir de las fauces identificadoras y reductoras de las concepciones del poder. Quizás el deseo tenga un papel relevante que cumplir, al formular rupturas con aquello que vivimos, con el presente-futuro llave de la reproducción del sistema. Así, una posibilidad real de reconfiguración de la semiótica puede ser aquella que parte del rastreo de los restos de deseo escondidos en lo cotidiano bajo otras formas.

Fredric Jameson propone el concepto utopología como una inversión estructural de la genealogía, en la búsqueda de las precondiciones (que no causas) necesarias para que algo acontezca. La utopología intentaría historizar el presente, es decir, analizarlo como parte de un proceso histórico de cambios. Los trazos del presente pueden imaginarse como componentes de un sistema futuro diferente, en el que quizás haya un cambio de valencias (Jameson, 2013, 494). Esta estrategia podría servir, al menos, para fortalecer los músculos de la imaginación, dejando una abertura para la irrupción del deseo, y sería un trabajo previo a toda acción política transformadora.

Lo que nos queda pues son las distorsiones y las derivas con las que imaginamos una salida del sistema que no pase por una fase de total destrucción. Pero el hecho de trabajar con distorsiones y restos no implica nada más que la posibilidad de que algo acontezca, pues allí donde todo encaja, nada sucede.

De ese modo la utopía surge como una ruptura, un punto de flexión entre la identidad y la diferencia, entre lo individual y lo colectivo, permitiendo la posibilidad de apertura de una fisura, un flash, una distorsión a partir de la cual pueda aparecer una huella de otra realidad, que solo entonces parecería algo virtual -aquello imposible-, amenazando con tomar cuerpo colectivo, en conformación con las subjetividades.

La irrupción del deseo en tanto catexis libidinales inconscientes procura encontrar el espacio donde poder tomar forma consciente, un lugar a medio camino entre el interés de las subjetividades y el deseo inconsciente, entre el pasado y el futuro, un hueco por donde pueda presentarse el por-venir.

La utopía no es una identidad que se nos opone, y su inteligibilidad no es la nuestra, pero la marca de la utopía es lo cotidiano. Tout autre est tout autre, dice Jacques Derrida, dejando entrever que la diferencia más absoluta, la alteridad radical, no está solo en un lugar alejado e inalcanzable, sino que también habita lo cotidiano (Derrida, 1999, 110 y ss). La necesidad de rehabilitar el deseo va en esa dirección y creemos que es una herramienta imprescindible para la conformación (colectiva) de otras subjetividades (diferenciales), para llegar por lo menos a concebir, aun momentáneamente, como en un pestañeo, los rastros y huellas de otra realidad imposible que aguarda para entrar en conformación con nuestra subjetividad, y así poder aterrizar en nuestro aquí y ahora. 


\section{Referencias bibliográficas}

Arrighi, Giovanni (1994): El largo siglo XX, Akal, Madrid.

Bloch, Ernst (1977): El principio esperanza. Vol. I, Aguilar, Madrid.

Derrida, Jacques (1999): Donner (la) mort, Galilée, Paris.

Derrida, Jacques (2003): Papel máquina, Trotta, Madrid.

Frye, Northrop (1982): "Diversidad de utopías literarias", in Manuel, Frank E.: Utopías y pensamiento utópico, Madrid, Espasa Calpe.

Fumagalli, Andrea (2010): Bioeconomía y capitalismo cognitivo, Traficantes de sueños, Madrid.

Jameson, Fredric (2009): Arqueologías del futuro, Akal, Madrid.

Jameson, Fredric (2013): Valencias de la dialéctica, Eterna cadencia, Buenos Aires.

Lazzarato, Maurizio (2013): La fábrica del hombre endeudado, Amorrortu, Buenos Aires.

Marazzi, Christian (1998): El sitio de los calcetines, Akal, Madrid.

Marazzi, Christian (2014): Capital y lenguaje, Tinta limón, Buenos Aires.

Suvin, Darko (1984): Metamorfosis de la ciencia ficción, FCE, México D.F. 
